\title{
ATCP: Improving TCP performance over mobile wireless environments
}

\author{
Ajay Kr Singh \\ Dept of Computer Science \& Engg \\ IIT Bombay \\ aksingh@cse.iitb.ac.in
}

\author{
Sridhar Iyer \\ School of Information Technology \\ IIT Bombay \\ sri@it.iitb.ac.in
}

\begin{abstract}
Transmission Control Protocol (TCP) is known to suffer from performance degradation in mobile wireless environments. This is because such environments are prone to packet losses due to high bit error rates and mobility induced disconnections. TCP interprets packet losses as an indication of congestion and inappropriately invokes congestion control mechanisms, leading to degraded performance.

While there are several proposals to optimize TCP in the presence of high bit error rates and mobility, they focus mainly on scenarios where the TCP sender is a fixed host. In this paper we propose ATCP, an approach which mitigates the of degrading effect of host mobility on TCP performance for two-way data transfers, i.e. scenarios where the TCP sender is a mobile host, in addition to scenarios where the TCP sender is a fixed host.

ATCP uses network layer feedback in terms of disconnection and connection signals, to modify the congestion control mechanisms of TCP, thereby achieving enhanced throughput in mobile wireless environments. We have compared ATCP with 3-dupacks (3DA) [10], Freeze TCP [4] and TCP Reno, by simulations using ns-2. We show that ATCP achieves an improvement of up to $40 \%$ over TCP Reno in WLAN environments and up to $150 \%$ in WWAN environments in both directions of data transfer.
\end{abstract}

Index Terms-TCP adaptation, Mobility, Wireless Networks, Network feedback, Network simulation, Performance study.

\section{INTRODUCTION}

Transmission Control Protocol (TCP) [11] is a reliable, connection-oriented, full-duplex, transport protocol widely used in wired networks. TCP's flow and congestion control mechanisms are based upon the assumption that packet loss is an indication of congestion. While this assumption holds in wired networks, it does not hold in the case of mobile wireless networks. As shown in figure 1, a typical wireless mobile network has mobile hosts (MH) connected to base stations (BS) over wireless links. The base stations are inter-connected via wired network. Other fixed hosts $(\mathrm{FH})$ may also be part of the wired network. In such networks, packet losses also occur due to bad wireless channel conditions and intermittent disconnection introduced by mobility of the MH. TCP interprets these packet losses as congestion and invokes the congestion control mechanisms, which reduce the sending window multiplicatively (by half), thereby reducing the sending rate drastically. However, bad channel conditions and intermittent disconnections are typically transient phenomena. Hence the TCP congestion control response is inappropriate and also undesirable as it decreases the throughput, resulting in an underutilization of the network. While several approaches have been

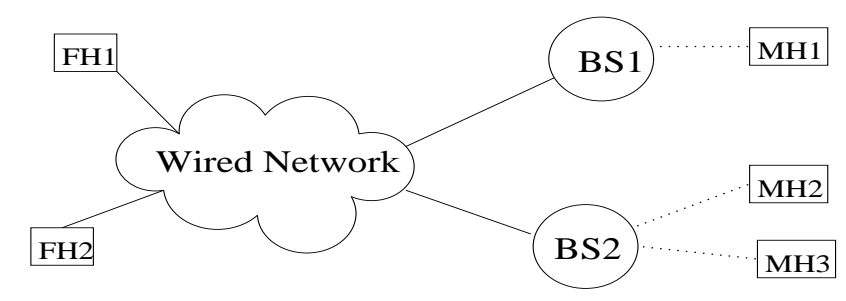

Fig. 1. A typical mobile wireless network

proposed for mitigating the effect of adverse channel conditions [1] [3] [5] [8] [9], there are few approaches [4] [6] [10], which tackle mobility induced disconnection. However, most of these schemes focus on scenarios where the TCP sender is a FH and do not perform well when the sender is a MH. Also, some of these approaches are vulnerable to scalability issues as they require per-flow support from the base station [9], or require modification to TCP at the $\mathrm{FH}$ [3]. In this paper we present ATCP, an adaptation to TCP, which alleviates the degrading effect of mobility on TCP performance. ATCP is designed for improving TCP performance in two-way data transfers, i.e., from $\mathrm{MH}$ to $\mathrm{FH}$ as well as from $\mathrm{FH}$ to $\mathrm{MH}$. ATCP requires modifications to TCP at the MH only, and uses feedback from network layer about the mobility status in terms of connection event and disconnection event signals, to modify the TCP congestion control mechanisms appropriately. ATCP uses these signals to appropriately freeze/continue ongoing data transfer and changes the action taken at RTO (Retransmission TimeOut) event, leading to enhanced TCP throughput. We have compared ATCP with 3-dupacks (3DA) [10], Freeze TCP [4] and TCP Reno, by simulations using ns-2. We show that ATCP achieves an improvement of up to $40 \%$ over TCP Reno in WLAN environments and up to $150 \%$ in WWAN environments in both directions of data transfer. This paper is organized as follows: Section II, discusses some related work and motivates the need for our approach. Section III describes our approach ATCP, Section IV presents the simulations results and Section $\mathrm{V}$ gives the conclusions.

\section{RELATED WORK}

There are several approaches for mitigating the effect of adverse channel conditions on TCP performance [1] [3] [5] [8] [9]. We focus on approaches that attempt to reduce the detrimental effect of host mobility on TCP perfor- 
mance [4] [6] [10]. Of these, we are primarily interested in approaches that require modifications only at the mobile host [4] [10]. Our approach ATCP falls into this category.

The 3-dupacks approach (3DA) [10] requires the network layer to provide information about ongoing mobility to the TCP layer at the mobile host (MH). After disconnection and upon subsequent reconnection, the $\mathrm{MH}$ sends three duplicate acknowledgements (dupacks) to the fixed host (FH). These dupacks cause the TCP sender at the FH to immediately enter the fast recovery phase, instead of waiting for its retransmission timer to expire. Thus this approach reduces the "idle period" of the TCP sender after the connection is re-established. However, the TCP sender at FH also reduces its slow start threshold (ssthresh) and congestion window (cwnd) parameters when it enters fast recovery phase. This side effect in turn reduces the throughput of the connection.

The Freeze TCP approach [4] requires the network layer at the $\mathrm{MH}$ to give an indication of impending disconnection. Upon receipt of such an indication, Freeze TCP at the MH sends a zero window advertisement to the FH. Upon reconnection, it uses 3DA [10] to restart transmission. The main drawback is that Freeze TCP requires the network layer to predict future disconnections. There is also the issue of how early should this prediction be made available to TCP at the MH. If it is available earlier than the RTT of the connection, Freeze TCP's action may lead to degraded performance. Since different connections may have different RTT values, this adds to the difficulty in accurate predictions.

In addition to 3DA and Freeze TCP, we have also compared the performance of ATCP with TCP Reno. Since TCP Reno is quite well-known and is described in standard text books [11], we omit discussing it here.

\section{ATCP MECHANISM}

ATCP is designed to improve TCP performance in wireless mobile networks in the presence of temporary disconnections caused by mobility. Unlike earlier work, ATCP improves the performance not only when the TCP sender is a fixed host (FH), but also when the TCP sender is a mobile host (MH). ATCP involves modifications to the network stack only at the $\mathrm{MH}$ and requires network layer feedback regarding the status of the connectivity.

ATCP assumes that the network layer sends a connection event signal to TCP when MH gets connected to the network and a disconnection event signal when the $\mathrm{MH}$ gets disconnected from the network. We believe that this is a reasonable assumption since such information is typically available with the network layer in wireless mobile networks (for instance, Mobile IP [7]). ATCP uses these signals to appropriately freeze/continue ongoing data transfer and changes the action taken at RTO (Retransmission TimeOut event), leading to enhanced TCP throughput.

In brief, the working of ATCP is as follows:

- MH to FH data transfer:

- Upon disconnection event, if the sending window is open (case 1, figure 2), ATCP does not wait for ACK for packets sent before disconnection, and cancels the retransmission timer (RTX). If the sending window is closed and it

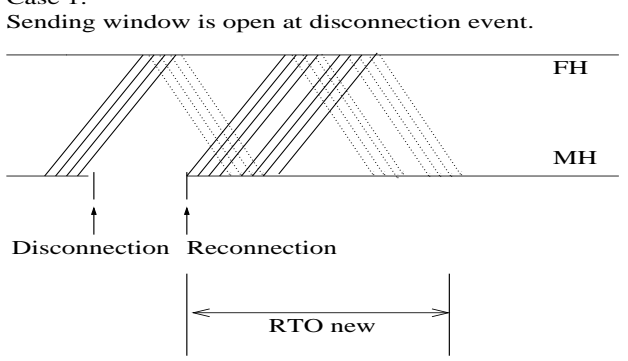

Case 2:

Sending window is closed at disconnection event. RTO event occurred during disconnection period.

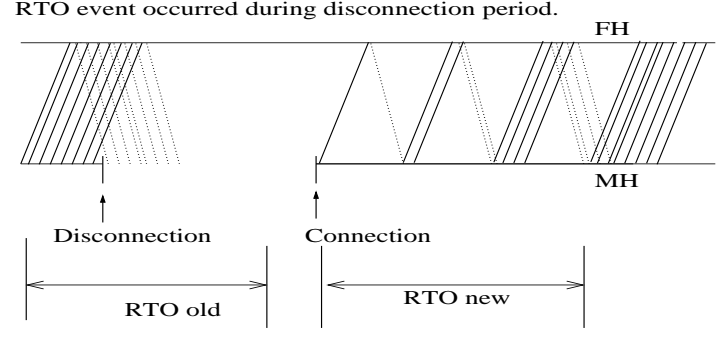

Case 3:

Sending window is closed at disconnection event.

No RTO event during disconnection period.

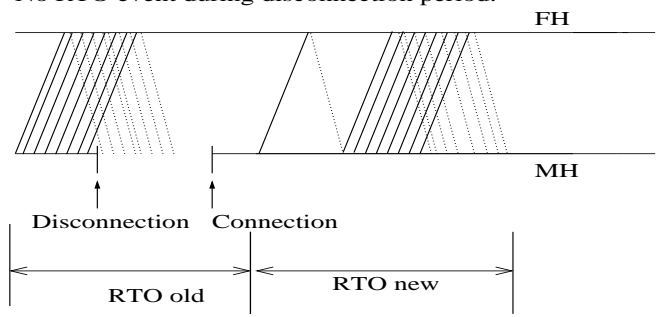

Fig. 2. ATCP behaviour under different disconnection scenarios

was waiting for ACKs, ATCP does not cancel RTX but waits for the occurrence of an RTO event.

- Upon connection event, if the sending window is open, ATCP sends data and sets a new RTX. Since ACKs are cumulative, the ACK for the new data also acknowledges the data sent before disconnection. If the sending window is closed and RTO has occurred, ATCP retransmits. If RTO has not occurred, ATCP waits for an RTO event.

- Upon RTO event, ATCP checks whether a disconnection has occurred. If so (case 2, figure 2), ATCP instead of reducing ssthresh (typical TCP behavior), sets ssthresh to the value of $c w n d$ at the time of disconnection and sets cwnd to one. If MH is connected to the network (case 3, see figure 2), ATCP retransmits the lost packet without modifying ssthresh or cwnd parameters.

These actions enable ATCP to quickly regain the cwnd value prior to disconnection, thus reducing under utilization of the available link capacity.

- FH to MH data transfer: ATCP delays the ACK for the last two bytes by $\mathbf{d}$ milliseconds, (at most 500 milliseconds [12]).

- Upon disconnection event, the network connectivity status is updated. 
- Upon connection event, ATCP ACKs the first of these bytes with zero window advertisement (ZWA) and ACKs the second byte with a full window advertisement (FWA). TCP at FH will process these ACKs as they have a higher sequence number [12] than all previous ACKs. ZWA causes TCP sender at FH to freeze its RTX, without reducing its $c w n d$. FWA results in the TCP sender at FH retransmitting all unacknowledged packets with no reduction in $c w n d$.

These action prevent the TCP at FH from taking congestion control measures when packets are lost due to $\mathrm{MH}$ being disconnected.

The ATCP algorithm is presented below.

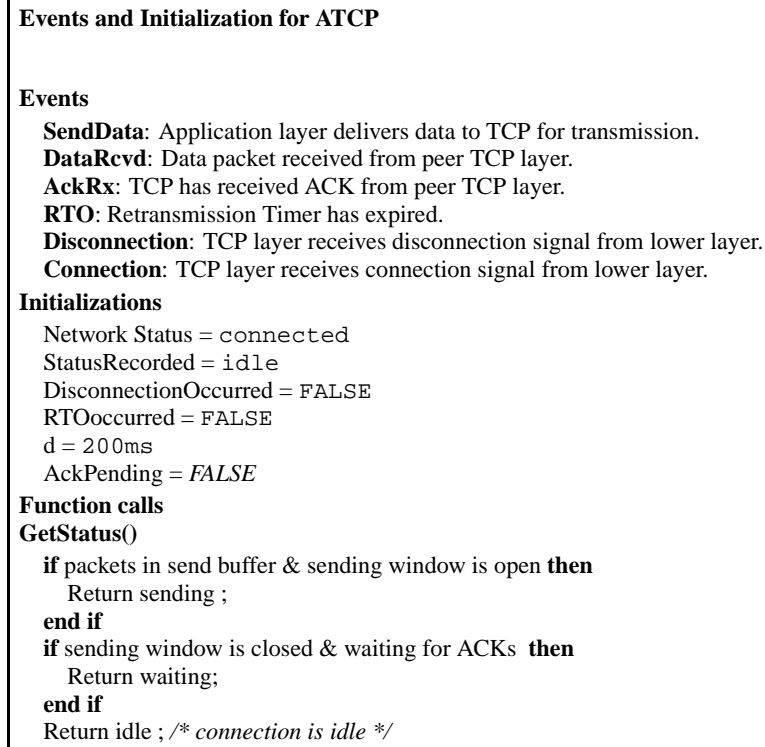

\section{Simulations}

We implemented ATCP, 3DA [10] and Freeze TCP [4] in the network simulator ns-2.1b8a [13]. TCP Reno is already implemented in this ns-2 distribution. We also modified MobileIP [7] in ns-2 for providing mobility information to the TCP Agent. Mobility of the MH is simulated by maintaining a variable, NetworkStatus, in TCP Agent whose value changes from Connected to Disconnected or vice versa, as determined by a disconnection timer handler. The disconnection timer can be configured to alternately connect and disconnect to simulate disconnection.

The simulation scenario is shown in figure 3. An FTP application simulated a large data transfer, with packet size 1000 bytes, and throughput of TCP connections was measured. For the disconnection duration, values ranging from $50 \mathrm{~ms}$ to $4 \mathrm{~s}$ were chosen. Typically smaller values occur in wireless LANs and larger values occurs in wireless WANs. The disconnection frequency was chosen as 10 seconds, indicating moderate to high mobility. The round trip time (RTT) was chosen as $5 \mathrm{~ms}$ for representing a wireless LAN (WLAN) and 700ms for representing a wireless WAN (WWAN). The link capacity was taken as $10 \mathrm{Mbps}$ for the $5 \mathrm{~ms}$ RTT (WLAN), and as $100 \mathrm{Kbps}$ for the 700ms RTT (WWAN). The capacity of both the links, i.e., from

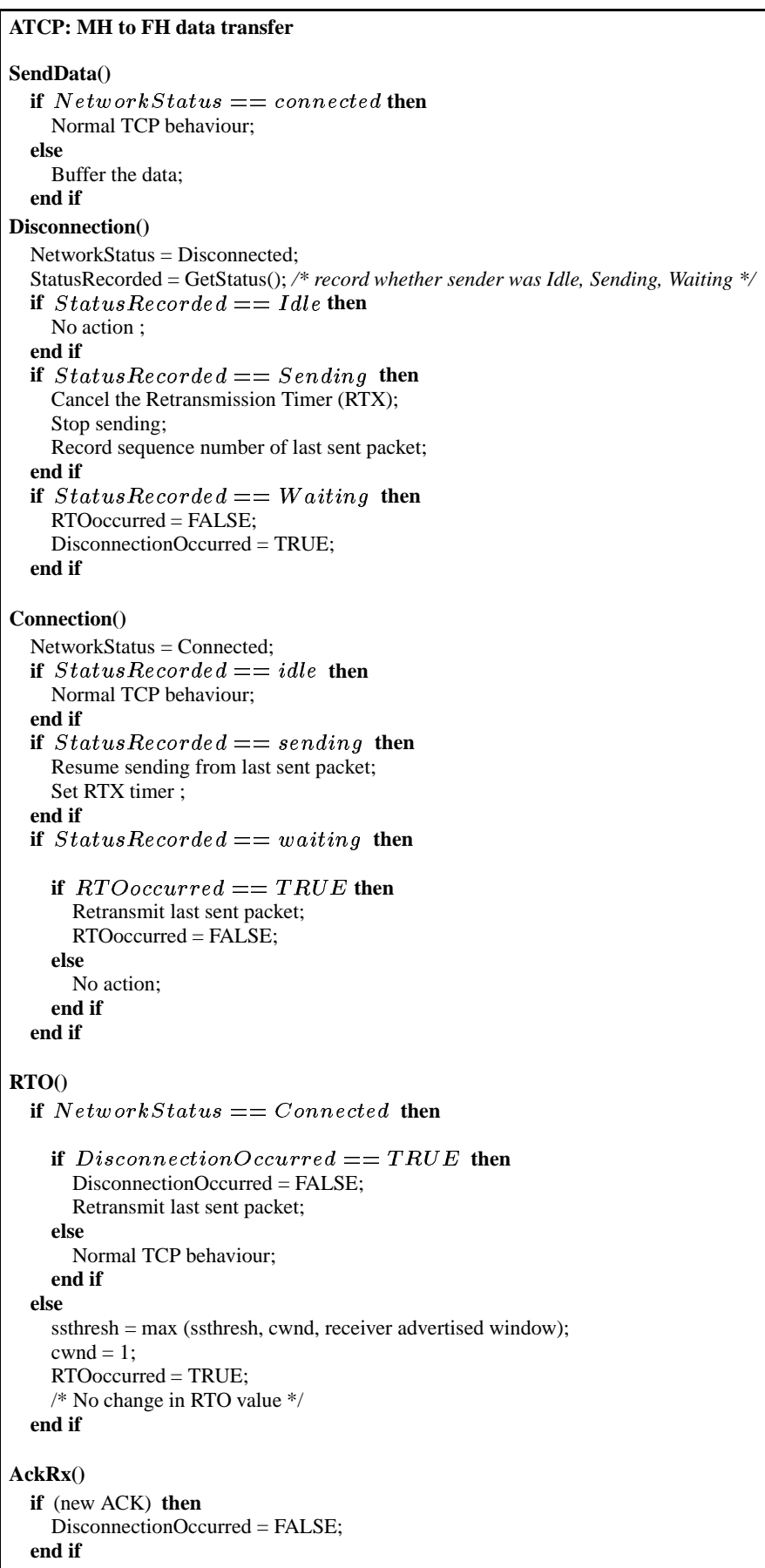

$\mathrm{FH}$ to $\mathrm{BS}$ and from $\mathrm{BS}$ to $\mathrm{MH}$ were maintained to be equal to avoid packet losses due to buffer overflow in routers. The simulations were carried out for 100s for WLAN environment and for 1000 s for WWAN environment.

\section{A. Analysis \& Comparison}

Observations resulting from the simulation are as follows:

- MH to FH data transfer: The 3DA and Freeze TCP approaches do not mention any actions specific to the MH being the TCP sender, hence we compared ATCP only with TCP Reno in this case. As can be seen from figures 4, 5:

- ATCP shows more enhancement in throughput as compared to TCP Reno, as the disconnection interval in- 
